\title{
Sliding Mode Control with Auto-Tuning Law for Maglev System
}

\author{
Lingling Zhang ${ }^{1}$, Zhizhou Zhang ${ }^{2 *}$, Zhiqiang Long ${ }^{2}$, Aming $\mathrm{Hao}^{2}$ \\ ${ }^{1}$ College of Mathematics and Econometrics, Hunan University, Changsha, China \\ ${ }^{2}$ College of Mechatronics Engineering and Automation, National University of Defense Technology, \\ Changsha, China \\ E-mail:zzz336@126.com \\ Received September 5, 2009; revised September 27, 2009; accepted October 4, 2009
}

\begin{abstract}
This paper presents a control strategy for maglev system based on the sliding mode controller with auto-tuning law. The designed adaptive controller will replace the conventional sliding mode control (SMC) to eliminate the chattering resulting from the SMC. The stability of maglev system is ensured based on the Lyapunov theory. Simulation results verify the effectiveness of the proposed method. In addition, the advantages of the proposed controller are indicated in comparison with a traditional sliding mode controller.
\end{abstract}

Keywords: Sliding Mode Control, Maglev System, Lyapunov Theory, Auto-Tuning

\section{Introduction}

Maglev (Magnetic Levitation) train is a late-model railway vehicle with many good performances such as high speed, comfort, low environmental pollution, low energy consumption and so on. Lots of countries have started up the engineering study of maglev train $[1,2]$.

The dynamic response of vehicle/guideway system affects the running safety, ride comfort and system costs heavily, which are crucial factors for maglev train commercial application [3,4]. Due to open-loop instability and inherent nonlinearities associated with a voltage-controlled magnetic levitation system, a feedback control is necessary to achieve a stable operation. Recently, quite a few control strategies have been developed and applied widely in the industrial field of maglev technology, such as bang-bang control [5], adaptive non-smooth control [6], hybrid control [7] and $H_{\infty}$ control method [8], etc.

Sliding mode control is a powerful robust approach for controlling the nonlinear dynamic systems [9]. The advantage of sliding mode control is robustness against parameter matched uncertainties and external disturbance and so on. In general, vehicle-guide vibration of maglev system is easily subjected to external disturbance. Even though the approach of sliding mode control is one of potential control candidates for maglev system, it has some limitations such as discontinuous control law and chattering action, which lead to the appearance of input chattering, the high-frequency plant dynamics and unforeseen instability in real application. In order to alleviate the high-frequency chattering, control researchers have proposed many strategies, such as the boundary layer technique $[10,11]$ and parameter identification mechanism with self-tuning [12,13]. In [14-16], using a modified hyperbolic tangent function as the activation function, the laws for tuning boundary layer thickness and control gain were proposed.

In this paper, the sliding mode control technique with auto-tuning law is applied to a voltage-controlled magnetic levitation system. The task of the control system is to dynamically regulate control voltage which drives the magnet to adjust the magnetic force to maintain a desired gap. Firstly, to simplify the mathematical model of maglev system, we discuss a 4-D maglev system via alternating physical variables of maglev system. In the following, with regard to this maglev system, four error variables are chosen to define the switching surface, and then a traditional sliding mode controller is designed accordingly [17]. In order to eliminate high-frequency control and chattering around the sliding surface, an auto-tuning neuron is introduced as an adaptive controller to guarantee the convergence of all states for maglev system. Simulations results show the control performance of our proposed method. 
The paper is organized as follows: In Section 2, we give a mathematical model of maglev system, and then choose four error variables to determine the sliding surface. In Section 3, we design a general sliding mode controller and a systematic sliding mode controller with self-tuning law respectively. Simulation results will be given to validate the effectiveness of our proposed controller in Section 4. Conclusions are drawn in Section 5.

\section{Sliding Surface Design of the Maglev System}

The maglev system is a complicated system with machinery, controlling and electromagnetic elements integrated together. Figure 1 shows its working elements.

According to [3], $m \mathrm{~m}$ denotes the weight of the electromagnet; and $z$ represents the absolute displacement of the electromagnet in the vertical. $R$ is the resistance of the electromagnet. $I$ and $V(t)$ are current and voltage of the electromagnet winding, respectively. The control current $I$ is driven by control voltage $V(t)$ to maintain the air gap $z$ at its nominal value. Define $y=\left[y_{1}, y_{2}, y_{3}\right]^{T}=[z, \dot{z}, I]^{T}$, then dynamical and electromagnetic equations of the system are given as

$$
\begin{aligned}
& \dot{y}_{1}=y_{2}, \\
& \dot{y}_{2}=g-\frac{k}{m} \frac{y_{3}^{2}}{y_{1}^{2}}, \\
& \dot{y}_{3}=\frac{y_{2} y_{3}}{y_{1}}-\frac{R y_{1} y_{3}}{2 k}+\frac{y_{1}}{2 k} V .
\end{aligned}
$$

where $k=\frac{1}{4} \mu_{0} N^{2} S_{0}, \mu_{0}$ is the magnetic permeability in vacuum, $N$ is the number of turns of coil, and $S_{0}$ is the effective pole area of electromagnet.

Note that this electromagnetic suspension system is unstable without control voltage $V$. In closed-loop system, gap sensor measures the relative interval between electromagnet and guideway while accelerometer measures the absolute kinetic acceleration of the electromagnet. Based on feedback signals from sensors, the controller can generate certain control voltage according to control algorithm. With the calculated control voltage, the electromagnet can produce suitable electromagnetic force to keep itself suspending under the guideway stably.

In order to simplify the original nonlinear system (1), we choose the variable

$X^{*}=\left[x_{1}^{*}, x_{2}^{*}, x_{3}^{*}, x_{4}^{*}\right]^{T}=\left[z, \dot{z}, I / Z,(I / Z)^{\prime}\right]^{T}=\left[y_{1}, y_{2}, y_{3} / y_{1},\left(y_{3} / y_{1}\right)^{\prime}\right]^{T}$, $x_{3}^{*}$ represents electromotive force of the electromagnet winding which has actual physical meanings. Then the model (1) can be transformed as following:

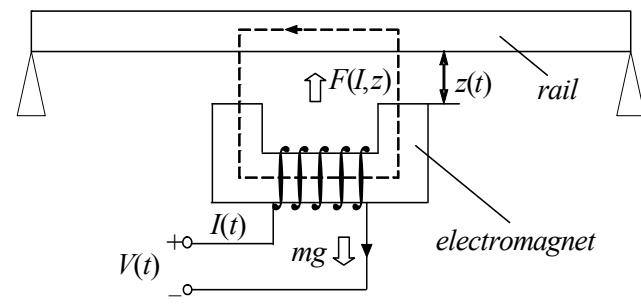

Figure 1. Structure of the maglev system.

$$
\begin{aligned}
& \dot{x}_{1}^{*}=x_{2}^{*}, \\
& \dot{x}_{2}^{*}=g-\frac{k x_{3}^{* 2}}{m}, \\
& \dot{x}_{3}^{*}=x_{4}^{*}, \\
& \dot{x}_{4}^{*}=-\frac{R}{2 k}\left(x_{1}^{*} x_{4}^{*}+x_{2}^{*} x_{3}^{*}\right)+\frac{1}{2 k} V^{\prime},
\end{aligned}
$$

where $V^{\prime}\left(x_{1}^{*}, x_{2}^{*}, x_{3}^{*}, x_{4}^{*}\right)$ is the derivatives of $V\left(x_{1}^{*}, x_{2}^{*}, x_{3}^{*}, x_{4}^{*}\right)$. And the values of the state variables at the balance point are $X_{0}^{*}=\left[z_{0}, 0, \sqrt{m g / k}, 0\right]$, where $z_{0}$ is a nominal value of the air gap $z$.

Moving the equilibrium to the origin, letting $X=\left[x_{1}, x_{2}, x_{3}, x_{4}\right]^{T}$, where $x_{1}=x_{1}^{*}-z_{0}, x_{2}=x_{2}^{*}$, $x_{3}=x_{3}^{*}-\sqrt{m g / k}, x_{4}=x_{4}^{*},(2)$ becomes

$\dot{x}_{1}=x_{2}$,

$\dot{x}_{2}=-\frac{k x_{3}^{2}}{m}-2 \sqrt{k g / m} x_{3}$,

$\dot{x}_{3}^{*}=x_{4}^{*}$,

$\dot{x}_{4}^{*}=-\frac{R}{2 k}\left(\left(x_{1}+z_{0}\right) x_{4}+\left(x_{3}+\sqrt{m g / k}\right) x_{2}\right)+\frac{1}{2 k} U$

where

$$
\begin{gathered}
U\left(x_{1}, x_{2}, x_{3}, x_{4}\right)=V^{\prime}\left(x_{1}^{*}, x_{2}^{*}, x_{3}^{*}, x_{4}^{*}\right)= \\
V^{\prime}\left(x_{1}+z_{0}, x_{2}, x_{3}+\sqrt{m g / k}, x_{4}\right)
\end{gathered}
$$

The system output is $Y=[1,0,0,0] X=x_{1}$. For convenience, denote $f_{1}=-\frac{k x_{3}^{2}}{m}-2 \sqrt{\frac{k g}{m} x_{3}}$, $f_{2}=-\frac{R}{2 k}\left(\left(x_{1}+z_{0}\right) x_{4}+\left(x_{3}+\sqrt{m g / k}\right) x_{2}\right)$.

Even now, a control scheme is presented to stabilize the states of maglev system. Our control objective is to stabilize all states of (3) to zero, that is to design control input to maintain the air gap $z$ at its nominal value.

Similar to linear system, the sliding mode control of nonlinear system consists two relatively independent parts: firstly confirm that the motion on the sliding surface is globally stable, and then design a sliding control- 
ler which causes the trajectories of the system to reach the sliding surface in finite time.

Denote the error variables as

$$
\begin{aligned}
& e_{1}=x_{1}, \\
& e_{2}=x_{2}, \\
& e_{3}=f_{1}=-\frac{k x_{3}^{2}}{m}-2 \sqrt{\frac{k g}{m}} x_{3}, \\
& e_{4}=\dot{f}_{1}=-2\left(\frac{k x_{3}}{m}+\sqrt{\frac{k g}{m}}\right) x_{4} .
\end{aligned}
$$

Then a switching surface is defined as $s=c_{1} e_{1}+c_{2} e_{2}+c_{3} e_{3}+e_{4}$, where $c_{1}, c_{2}, c_{3}$ are selected to be positive constant numbers such that the polynomial $\lambda^{3}+c_{3} \lambda^{2}+c_{2} \lambda+c_{1}$ is Hurwitz. The error dynamics in sliding mode are thus asymptotically stable.

The second step is to design a sliding controller such that the sliding surface approaches 0 . And the control input $U$ can be formulated as

$$
\begin{array}{r}
U=-\left[\frac{1}{2 k} \frac{\partial f_{1}}{\partial f_{3}}\right]^{-1}\left\{c_{1} x_{2}+c_{2} f_{1}+c_{3} \frac{\partial f_{1}}{\partial f_{3}} x_{4}+\right. \\
\left.\frac{d}{d t}\left[\frac{\partial f_{1}}{\partial f_{3}}\right] x_{4}+\frac{\partial f_{1}}{\partial f_{3}} x_{2}+\beta \operatorname{sign}(s)\right\}
\end{array}
$$

where $\beta$ is a positive constant.

Theorem 1. Under the control (5), all states of (4) will asymptotically converge to zero.

Proof. Select a Lyapunov function candidate: $V=s^{2} / 2$. Differentiating $V$ and substituting (4)-(5) yield,

$$
\begin{aligned}
& \dot{V}=s \dot{s}=s\left[c_{1} \dot{e}_{1}+c_{2} \dot{e}_{2}+c_{3} \dot{e}_{3}+\dot{e}_{4}\right] \\
& =s\left[c_{1} x_{2}+c_{2} f_{1}+c_{3} \frac{\partial f_{1}}{\partial f_{3}} x_{4}+\frac{d}{d t}\left(\frac{\partial f_{1}}{\partial f_{3}}\right) x_{4}+\frac{\partial f_{1}}{\partial f_{3}}\left(f_{2}+\frac{1}{2 k} U\right)\right] \\
& =-s \cdot \beta \operatorname{sign}(s)=-\beta|s| \leq 0
\end{aligned}
$$

For the controller (5), inequality (6) implies that the system can reach the surface, $s=0$, in finite time. $\beta$ represents the amplitude of related to the sign-function, which has a relationship to the velocity of reaching the sliding surface. Since $c_{1}, c_{2}, c_{3}$ are selected to be positive constant numbers such that the polynomial $\lambda^{3}+c_{3} \lambda^{2}+c_{2} \lambda+c_{1}$ is Hurwitz, $e_{1}, e_{2}$ and $e_{3}$ will all converge to zero from any initial conditions. On the ideal sliding mode $s=0$, that is $e_{4}=-c_{1} e_{1}-c_{2} e_{2}-c_{3} e_{3}, e_{4}$ will converge to zero too.

Then, we explain the vector $X=\left[x_{1}, x_{2}, x_{3}, x_{4}\right]^{T}$ will converge to 0 if $E \rightarrow 0$. From (4), we get that $e_{1}=x_{1}, e_{2}=x_{2}$ and $e_{3}=f_{1}$ are stabilized. In practical application, the current $I$ and the air gap $z$ are positive, so $x_{3}^{*}(=I / z)$ must be positive from Equation (2), then $x_{3}\left(=x_{3}^{*}-\sqrt{m g / k}\right)$ should be larger than in Equation (3). It follows that $x_{3}$ has to converge to zero from the stability of $e_{3}\left(=f_{1}=-\frac{k x_{3}^{2}}{m}-2 \sqrt{\frac{k g}{m}} x_{3}\right)$. Similarly $x_{4}$ will converge to zero if $e_{4}$ is stabilized. Therefore, under the controller (5), the state variables of system (3) will all converge to the equilibrium point.

\section{Adaptive Sliding Mode Controller Design}

In order to eliminate the chattering typically found in conventional sliding mode control, we utilize the boundary layer technique $[14,15,18]$. If the control gain constant and the width of the boundary layer are fixed numbers, there is no guarantee for fast convergence. So we introduce an auto-tuning neuron to be the direct adaptive neural controller. The structure of an auto-tuning neuron can be mathematically expressed as $[14,15,18]: \sigma=E-\varphi$, where E represents the external input of neuron; $\varphi$ denotes threshold of bias, and $\sigma$ is the internal state of neuron.

The auto-tuning sliding mode control law for system (3) is

$$
U=-\left[\frac{1}{2 k} \frac{\partial f_{1}}{\partial f_{3}}\right]^{-1}\left[c_{1} x_{2}+c_{2} f_{1}+c_{3} \frac{\partial f_{1}}{\partial f_{3}} x_{4}+\frac{d}{d t}\left[\frac{\partial f_{1}}{\partial f_{3}}\right] x_{4}+\frac{\partial f_{1}}{\partial f_{3}} f_{2}\right]+\phi(\sigma),(7)
$$

where $\phi(\sigma)$ is a modified hyperbolic tangent function,

$$
\phi(\sigma)=\frac{a[1-\exp (-b \cdot \sigma)]}{1+\exp (-b \cdot \sigma)},
$$

where $a$ is the saturated level; and $b$ is the slope value. Obviously, the shape of the nonlinear saturated function is governed by the values of both $a$ and $b$. Figure 2 gives the plot of (8), where two adjustable parameters $a$ and $b$ influence mainly the output range and the curve shape of the activation function. A larger $b$ corresponds to a narrower boundary layer. Let $\theta=[a, b, \varphi]^{T}$ represent the vector of adjustable parameters.

In the following we will adjust $\theta$ to achieve the control objective. The auto-tuning law is designed as

$$
\begin{aligned}
& \dot{a}=-\eta e_{1} \operatorname{sign}\left(\frac{\partial x_{1}}{\partial U}\right)\left(\frac{1-\exp (-b \cdot \sigma)}{1+\exp (-b \cdot \sigma)}\right), \\
& \dot{b}=-\mu e_{1} \operatorname{sign}\left(\frac{\partial x_{1}}{\partial U}\right) a \sigma \\
& \dot{\varphi}=-\dot{\sigma}=\gamma e_{1} \operatorname{sign}\left(\frac{\partial x_{1}}{\partial U}\right) a b,
\end{aligned}
$$

where $\eta, \mu$ and $\gamma$ are positive constants used to ad- 


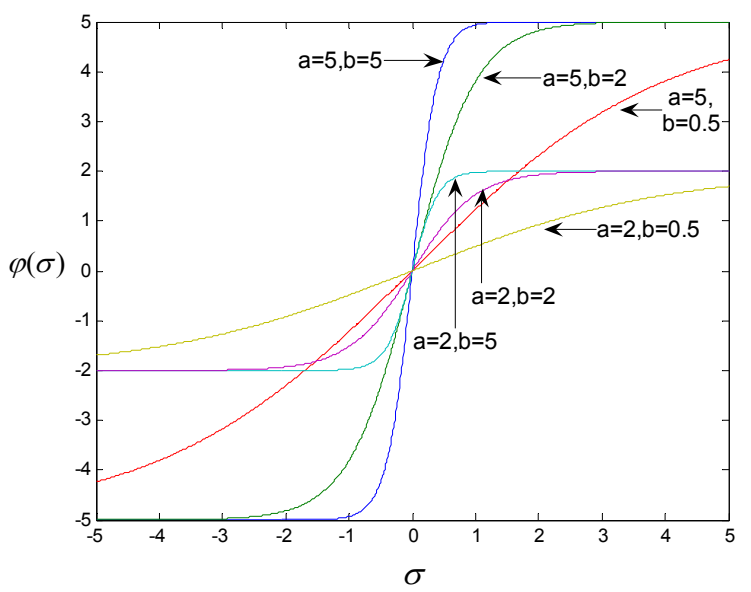

Figure 2. Modified activation function for different a and $b$.

just the convergence speed, and sign $\frac{\partial x_{1}}{\partial U}$ determines the direction of the search for $\theta$. The accurate value of $\frac{\partial x_{1}}{\partial U}$ is not important since the maglev system output monotonically increases as the control input to the controlled plant increases, that is system (3) is said to be positive responded [18]. Then the system direction is written as 1. Fortunately, there are many industrial process control systems that possess the property of positive-responded or negative-responded.

Theorem 2. Under the control (7) and (8), all states of the system (3) with the adaptation law (9) will aymtocally converge to 0 .

Proof. Consider the Lyapunov function candidate: $V=\frac{1}{2} e_{1}^{2}$. Differentiating V $[14,15,18]$, we have

$\frac{d V}{d t}=\frac{\partial V}{\partial e_{1}} \frac{\partial e_{1}}{\partial x_{1}} \frac{\partial x_{1}}{\partial U}\left(\frac{\partial U}{\partial a} \frac{\partial a}{\partial t}+\frac{\partial U}{\partial b} \frac{\partial b}{\partial t}+\frac{\partial U}{\partial \sigma} \frac{\partial \sigma}{\partial \varphi} \frac{\partial \varphi}{\partial t}\right)$

For convenience, denote the first term of the right hand side of (10) $V_{1}=\frac{\partial V}{\partial e_{1}} \frac{\partial e_{1}}{\partial x_{1}} \frac{\partial x_{1}}{\partial U} \frac{\partial U}{\partial a} \frac{\partial a}{\partial t}$, the second term and the third $V_{2}=\frac{\partial V}{\partial e_{1}} \frac{\partial e_{1}}{\partial x_{1}} \frac{\partial x_{1}}{\partial U} \frac{\partial U}{\partial b} \frac{\partial b}{\partial t}$, $V_{3}=\frac{\partial V}{\partial e_{1}} \frac{\partial e_{1}}{\partial x_{1}} \frac{\partial x_{1}}{\partial U} \frac{\partial U}{\partial \sigma} \frac{\partial \sigma}{\partial \varphi} \frac{\partial \varphi}{\partial t}$.

Substituting (7), (8) and (9) into (10) yield

$$
\begin{aligned}
& V_{1}=\frac{\partial V}{\partial e_{1}} \frac{\partial e_{1}}{\partial x_{1}} \frac{\partial x_{1}}{\partial U} \frac{\partial U}{\partial a} \frac{\partial a}{\partial t} \\
& =e_{1} \frac{\partial x_{1}}{\partial U} \frac{\partial}{\partial a}\left\{-\left[\frac{1}{2 k} \frac{\partial f_{1}}{\partial x_{3}}\right]^{-1}\left[c_{1} x_{2}+c_{2} f_{1}+c_{3} \frac{\partial f_{1}}{\partial x_{3}} x_{4}+\right.\right. \\
& \left.\left.\frac{d}{d t}\left(\frac{\partial f_{1}}{\partial x_{3}}\right) x_{4}+\frac{\partial f_{1}}{\partial x_{3}} f_{2}\right]+\frac{a[1-\exp (-b \cdot \sigma)]}{1+\exp (-b \cdot \sigma)}\right\} \dot{a} \\
& =-\eta e_{1}^{2}\left|\frac{\partial x_{1}}{\partial U}\right|\left(\frac{1-\exp (-b \cdot \sigma)}{1+\exp (-b \cdot \sigma)}\right)^{2} \leq 0
\end{aligned}
$$

Similarly, $V_{2}, V_{3}$ become respectively:

$$
\begin{aligned}
& V_{2}=\frac{\partial V}{\partial e_{1}} \frac{\partial e_{1}}{\partial x_{1}} \frac{\partial x_{1}}{\partial U} \frac{\partial U}{\partial b} \frac{\partial b}{\partial t} \\
& =-\mu e_{1}^{2}\left|\frac{\partial x_{1}}{\partial U}\right| \frac{2 \exp (-b \cdot \sigma)}{(1+\exp (-b \cdot \sigma))^{2}} a^{2} \sigma^{2} \leq 0 \\
& V_{3}=\frac{\partial V}{\partial e_{1}} \frac{\partial e_{1}}{\partial x_{1}} \frac{\partial x_{1}}{\partial U} \frac{\partial U}{\partial \sigma} \frac{\partial \sigma}{\partial \varphi} \frac{\partial \varphi}{\partial t} \\
& =-\gamma e_{1}^{2}\left|\frac{\partial x_{1}}{\partial U}\right| \frac{2 \exp (-b \cdot \sigma)}{(1+\exp (-b \cdot \sigma))^{2}} a^{2} b^{2} \leq 0
\end{aligned}
$$

where $e_{1} \frac{\partial x_{1}}{\partial U}$ represents system output error contributed by the control input [18].

Since $\frac{\partial V}{\partial t} \leq 0$ while $V>0$, according to the Lyapunov stability theory, $V$ will decrease to zero, so $e_{1} \rightarrow 0$, and $e_{i}(i=2,3,4)=0$. Therefore, similarly to the last paragraph in the proof of Theorem 1, the states of system (3) will all converge to zero.

\section{Simulation and Results}

In this section, we give the numerical simulation results of maglev system. The parameters and initial conditions used in the maglev system are given by $z_{0}=0.010 \mathrm{~m}$, $m=500 \mathrm{~kg}, R=4.4 \Omega, k=0.002, x(0)=(0.00458,0,1,0)^{T}$. The parameters and initial conditions of the controller for simulation are $c_{2}=22.25, \quad \eta, \mu, \gamma=0.1, \quad \beta=0.1$, $\theta(0)=(-0.1,0.2,0) T$, and time interval is $t=0.0001$,

As we know, the learning rate $\eta, \mu, \gamma$ play an important role in parameter learning process. For instance, a larger learning rate can accelerate the system response, but may also cause the system to have a larger overshoot. In the design of switching surface, $s=c_{1} e_{1}+c_{2} e_{2}+c_{3} e_{3}+e_{4}$, pole placement methodology [19] is often adopted to place the roots of the characteristic polynomial in desired region of the complex plane. That is, the control system denoted by the characteristic polynomial should have suitable damp, fast system response, short settling time and small overshoot. Therefore, $c_{1}, c_{2}, c_{3}$ are often chosen such that the three order polynomial has a pair of conjugate complex roots with negative real parts and a negative real root. When $c_{1}=21.75, c_{2}=22.25, c_{3}=8$, the roots of the characteristic polynomial are $-2.5 \pm 1 i$ and -3 .

Our control object is to design the control input to regulate the air gap to the desired value. Figure 3(a) and (b) represent the performance of state $x_{1}$ and the control input $U$ by using the traditional sliding mode con- 
trol, and it can be seen that the chattering around $x_{1}=0$ have occurred. The control input is regulated with high-frequency, which is not expected in industrial field. Figure 4(a) and (b) show the results by using our proposed method. Here, we choose $c_{1}=12, c_{2}=14, c_{3}=6$. The state $x_{1}$ is asymptotically controlled to 0 and the control input $U$ is modulated. In Figure 4(a), the regulation time of state $x_{1}$ is about 6 second and the overshoot is near to $0.6 \mathrm{~mm}$. In Figure 4(b), the overshoot of control input is reduced to 2 . These verify that better co ntrol performance of maglev system can be achieved by the proposed auto-tuning controller.

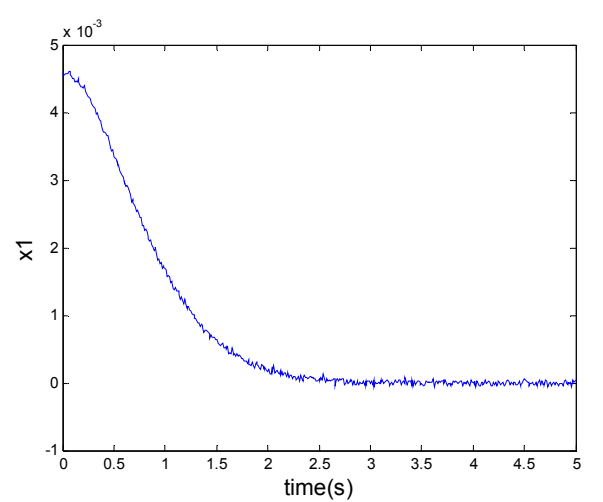

(a)

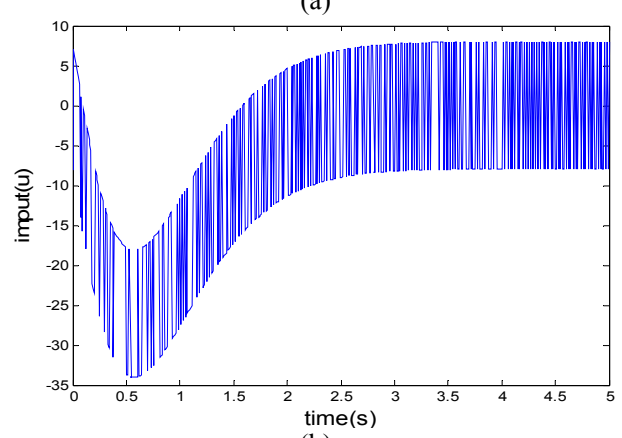

(b)

Figure3. (a-b) represent the control performance using the general sliding mode control.

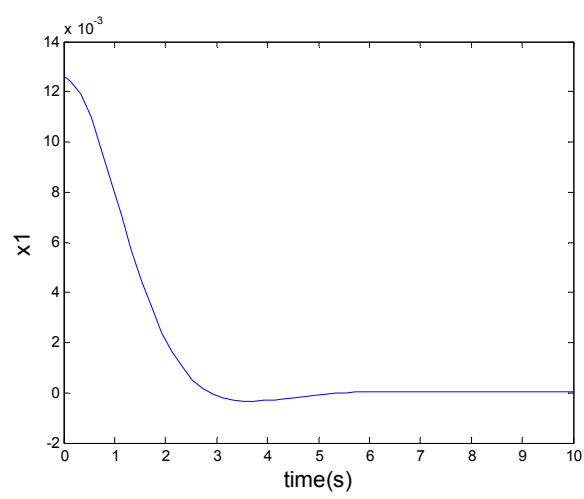

(a)

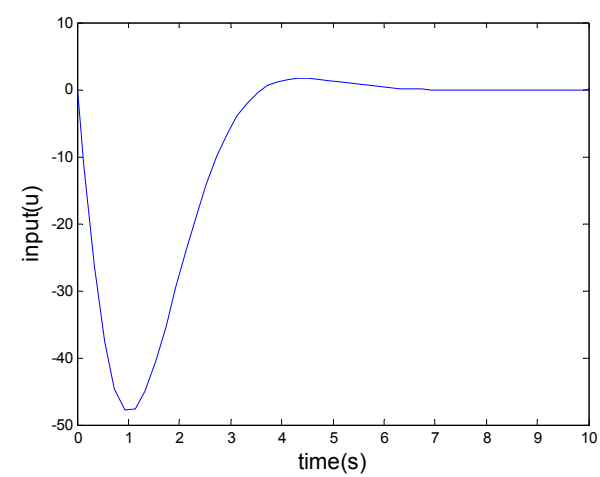

(b)

Figure 4. (a-b) show the results using the auto-tuning sliding mode control with control parameters $c_{1}=12, c_{2}=14, c_{3}=6$.

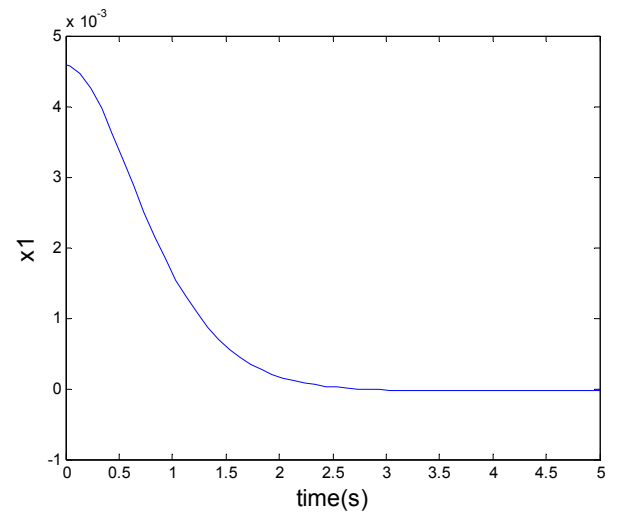

(a)

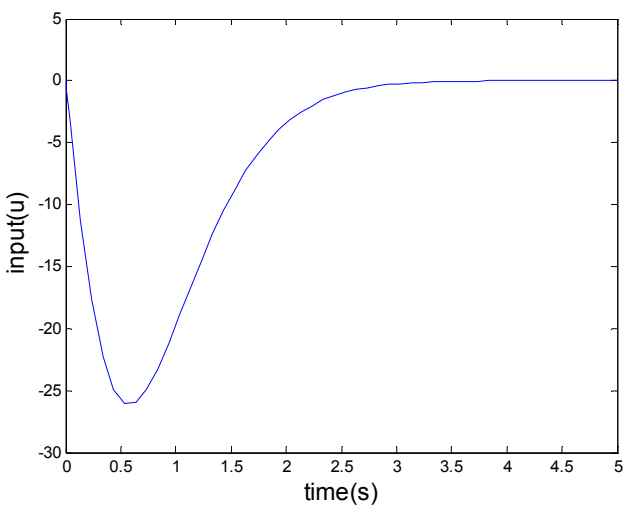

(b)

Figure 5. (a-b) show the results using the auto-tuning sliding mode control with control parameters $c_{1}=4, c_{2}=6, c_{3}=4$.

In Figure 4, the regulation time and overshoot is not satisfying, so here some new parameters $c_{1}=4$, $c_{2}=6, c_{3}=4$ by the proposed auto-tuning sliding mode control are chosen to accelerate system response and decrease the overshoot. In Figure 5(a), the convergence time of state $x_{1}$ slows to 3 second and the overshoot $x_{1}$ falls to 0 . In Figure 5(b), the overshoot of control input is reduced to 0 . It accords with the theoretical analysis. Based on the demand of practical application, 
we can regulate these parameters to get a better performance.

\section{Conclusions}

In this paper, we discuss the problem of vibration control of maglev problem via a sliding mode control approach. In order to eliminate the high control activity and chattering caused by the general sliding mode control, we present an auto-tuning law based on the Lyapunov stability theory to guarantee the convergence of the system states. Simulation results verify the proposed auto-tuning controller is better than the traditional sliding mode controller.

It should be pointed out that only three control parameters are chosen to achieve good performance for maglev system in this paper, but other factors also can be applied to sliding mode control in practice. Next plan is to combine with more state variables with elastic guideway conditions. The expected results should improve the suspension performance of flexible guideway with proposed control algorithms.

\section{References}

[1] Y. Yoshihide, F. Masaaki, T. Masao, et al, "The first HSST maglev commercial train in Japan," MAGLEV 2004 Proceedings, pp. 76-85, 2004.

[2] R. Goodall, "Dynamic and control requirements for EMS maglev suspension," MAGLEV 2004 Proceedings, pp. 926-934, 2004.

[3] H. Wang, J. Li, K. Zhang, "Non-resonant response, bifurcation and oscillation suppression of a non-autonom ous system with delayed position feedback control," Nonlinear Dynamic, Vol. 51, pp. 447-464, 2008.

[4] L. Zhang, L. Huang, Z. Zhang, "Stability and Hopf bifurcation of the maglev system with delayed position and speed feedback control," Nonlinear Dynamic, Vol. 57, pp. 197-207, 2009.

[5] C. Feng, W. U. Zhu, "Stochastic optimal control of strongly nonlinear systems under wide-band random ex- citation with actuator saturation," Acta Mechanica Solida Sinica, Vol. 21, No. 2, pp. 116-126, 2008.

[6] N. Taher, "A new fuzzy adaptive hybrid particle swarm optimization algorithm for non-linear, non-smooth and non-convexeconomic dispatch problem," Applied Energy,doi:10.1016/j.apenergy.2009.05.016, 2009.

[7] H. Tomohisa, M. Wassim, Y. Konstantin, "Neural network hybrid adaptive control for nonlinear uncertain impulsive dynamical systems," Nonlinear Analysis: Hybrid Systems, Vol. 2, pp. 862-874, 2008.

[8] V. Suplina, U. Shaked, "Robust H1 output-feedback control of systems with time-delay," Systems and Control Letters, Vol. 57, No. 3, pp. 193-199, 2008.

[9] B. Giorgio, F. Leonid, P. Alessandro, et al, "Modern sliding mode control theory," Berlin, Spinker, 2008.

[10] J. J. Slotine, W. Li, “Applied nonlinear control," Englewood Cliffs (NJ), Prentice-Hall, 1991.

[11] Y. J. Huang, T. C. Kuo, "Robust position control of DC servomechanism with output measurement noise," Electrical Engineering, Vol. 88, pp. 223-338, 2006.

[12] K. Furuta, "VSS type self-tuning control," IEEE Transactions on Industrial Electronics, 40, pp. 37-44, 1993.

[13] P. M. Lee, J. H. Oh, "Improvements on VSS type self-tuning control for a tracking controller," IEEE Transactions on Industrial Electronics, Vol. 45, pp. 319 $325,1998$.

[14] C. T. Chen, W. D. Chang, "A feedforward neural network with function shape autotuning," Neural Networks, Vol. 9, No. 4, pp. 627-641, 1996.

[15] W. D. Chang, R. C. Hwang, J. G. Hsieh, "Application of an auto-tuning neuron to sliding mode control," IEEE Transactions on Systems, Man, and Cybernetics-Part C, Vol. 32, pp. 517-529, 2002.

[16] T. C. Kuo, J. H. Ying, S. H. Chang, "Sliding mode control with self-tuning law for uncertain nonlinear systems," ISA Transactions, Vol. 47, pp. 171-178, 2008.

[17] X. Rong, Ä. O. ÄUmit, "Sliding mode control of a class of underactuated systems," Automatica, Vol. 44, pp. 233-241, 2008.

[18] X. Cui, K. G. Shin, "Direct control and coordination using neural networks," IEEE Transactions on Systems, Man, and Cybernetics, Vol. 23, No. 3, pp. 686-697, 1993.

[19] C. D. Richard, H. B. Rober, "Modern control systems," Addison Wesley Longman, 1993. 